EPJ Web of Conferences 71, 00055 (2014)

DOI: 10.1051/epjconf/20147100055

(C) Owned by the authors, published by EDP Sciences, 2014

\title{
An Overview of Recent Results from STAR
}

\author{
Bingchu Huang ${ }^{1, a}$ for the STAR Collaboration \\ ${ }^{1}$ Brookhaven National Laboratory, Upton, New York, 11973, USA
}

\begin{abstract}
The STAR experiment at the Relativistic Heavy Ion Collider has collected wealthy data in multiple collision systems at different beam energies. We present an overview of recent results from STAR. Results of anisotropic flows, heavy flavors and dileptons are reported.
\end{abstract}

\section{Introduction}

Relativistic Heavy Ion Collider (RHIC) provides an extreme condition of hot, dense and strongly interacting matter in heavy ion collisions, so called Quark Gluon Plasma (QGP). Studies of QGP properties require some unique probes. Elliptic flow which is defined as the second harmonic moment, $v_{2}$, of the Fourier decomposition of azimuthal anisotropy is an important observable for studying heavy ion collisions. The $v_{2}$ measurements were consistent to the predictions from hydrodynamics models [1] at low $p_{T}$, and this consistency breaks down at high $p_{T}$. The ideal hydrodynamics models predicts a continued rise with $p_{T}$ while the $v_{2}$ of mesons saturated at $2 \mathrm{GeV} / c$ and $3 \mathrm{GeV} / c$ for protons [2]. At intermediate $p_{T}\left(2<p_{T}<6 \mathrm{GeV} / c\right)$, a Number-of-Constituent Quark (NCQ) scaling of $v_{2}$ was suggested to explain the measured hadron $v_{2}$ [3]. This observation was used to provide clear evidence that the relevant degrees of freedom in the systems formed at top RHIC energy are quarks and gluons [4], it implies that this strongly-coupled parton medium, so called sQGP, behaves like a nearly perfect liquid and has partonic collectivity [5]. The interpretation also indicates that elliptic flow signature is response to the initial geometry of the system. The measurements of identified particles $v_{2}$ provide unmatched capability to systematically study the partonic collectivity and sQGP properties with recent STAR data. In 2012, RHIC for the first time collided two uranium beams. The highly deformed shape of uranium nucleus allows to study the initial geometry contribution to the elliptic flow.

Heavy quarks, charm and bottom, are produced by the hard process in the initial interactions with large momentum transfer before QGP formed. They have different interactions with the QGP compare to light quarks. Hence, the heavy quarks are ideal probes of the QGP properties. Experimentally, the decay products of heavy quark mesons and the heavy quarkonia like $\mathrm{J} / \psi$ are used for study heavy quark productions and interaction with medium. Also the elliptic flow of heavy quark serves as an important tool for studying collective behavior of nuclear matter in heavy ion collisions.

Dileptons are unique probes in the study of the hot and dense matter [6]. Because they are produced through out the whole evolution of a heavy-ion collision and have negligible final state interactions with created nuclear matter. The kinematics of dilepton production of different processes deter-

ae-mail: huangbc@bnl.gov

This is an Open Access article distributed under the terms of the Creative Commons Attribution License 2.0, which permits unrestricted use, distribution, and reproduction in any medium, provided the original work is properly cited. 


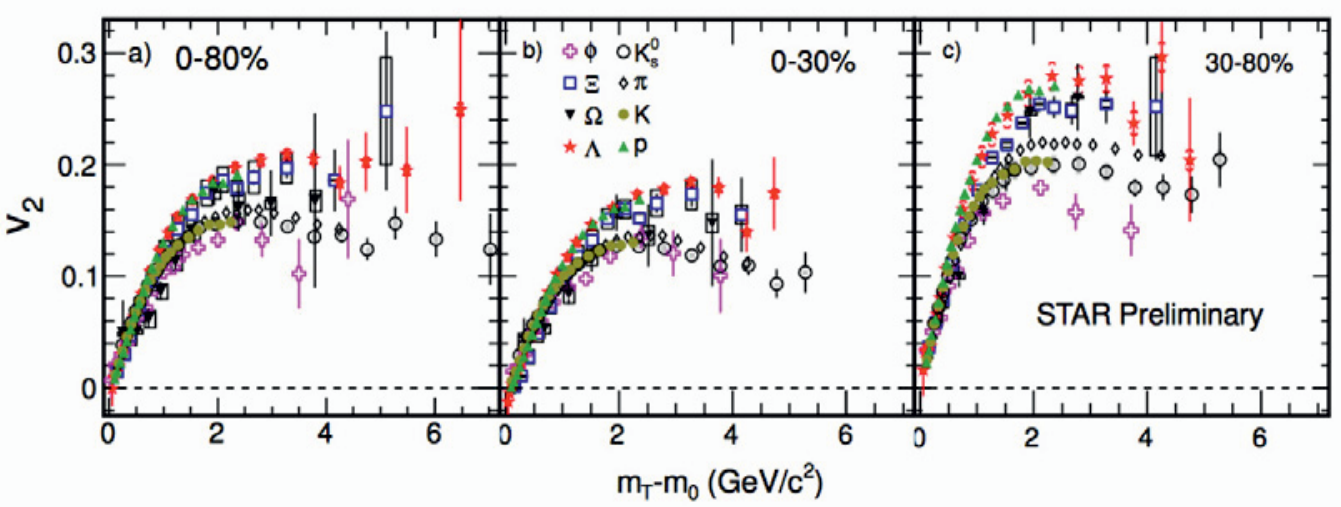

Figure 1. Identified particle $v_{2}$ vs. transverse kinetic energy $\left(m_{T}-m_{0}\right)$ in $\mathrm{Au}+\mathrm{Au} 200 \mathrm{GeV}$ for $0-80 \%$ minimum bias (a), $0-30 \%$ (b) and 30-80\% (c) centralities. Statistical error are shown as bars. Caps, open or shaded boxes are systematic uncertainties on different particles.

mined the distributions of invariant mass and transverse momentum of lepton pairs, therefore, we can selectively probe the subsequent stages of evolution with certain ranges in the dilepton invariant mass spectrum. In the high invariant mass range (HMR) $\left(M_{l l}>3 \mathrm{GeV} / c^{2}\right)$, dileptons are mainly from heavy quarkonia decays, such as $\mathrm{J} / \psi$ and $v$. It provides a way to study deconfinement and color screening features in the hot an dense medium [7]. In intermediate mass range (IMR) $\left(1.1<M_{l l}<3.0 \mathrm{GeV} / \mathrm{c}^{2}\right)$, the dilepton mass spectra are expected to be related to charm quark production and the thermal radiation of the QGP [10]. At higher center-of-mass energies, the dilepton production from charm quarks are increasing dramatically which is suitable for studying charm modification in medium. However, the thermal radiation contribution will become extremely hard to measure. In low mass range (LMR) $\left(M_{l l}<1.1 \mathrm{GeV} / c^{2}\right)$, the dilepton yields are dominated by light meson decays, such as the Dalitz decays of $\pi^{0}, \eta$, and leptonic decays of the $\rho, \omega$, and $\phi$ vector mesons. Any in-medium modifications of the spectral shape of these mesons may have possible link to the chiral symmetry restoration [9]. Especially, the $\rho$ meson is expected to be sensitive to the in-medium modifications since it has short lifetime $1.3 \mathrm{fm} / \mathrm{c}$ and strong coupling interactions to the $\pi \pi$ channel.

At SPS energies, both the CERES and NA60 observed low mass dilepton enhancement [11, 12], which could be explained as a broadening scenario with in-medium modified $\rho$ spectral function. Further more, NA60 di-muon measurement excluded a dropping mass scenario. However, the broadening scenario can not be able to describe the PHENIX results in most central collisions at RHIC energy. Systematically study of dilepton production in different energies can provide insight understanding of the in-medium modifications of vector mesons. With RHIC Beam Energy Scan (BES) program in 2010 and 2011, STAR can measure the dilepton in the low and intermediate mass ranges from top RHIC beam energies down to SPS energies. In this paper, selected STAR results of elliptic flow, heavy flavor and dileptons will be discussed.

\section{Elliptic flow at top RHIC energy}

The observations of the strong elliptic flow and the NCQ scaling of multi-strange hadrons are interpreted as a signature of partonic collectivity, a critical feature of the sQGP [3]. With the unmatched statistical dataset in $\mathrm{Au}+\mathrm{Au}$ at $200 \mathrm{GeV}$, we are able to test the NCQ scaling with high precision and 


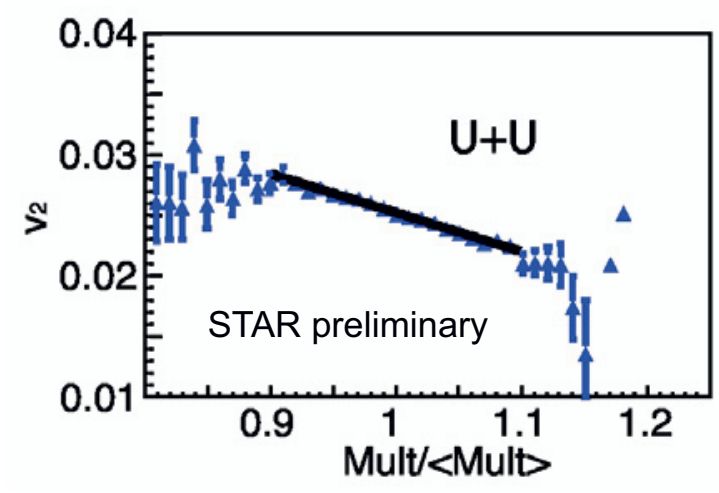

Figure 2. The $v_{2}$ of charged particles as a function of multiplicity divided by mean multiplicity in $0.1 \%$ ZDC centrality in $\mathrm{U}+\mathrm{U}$ collisions at $193 \mathrm{GeV}$. A linear fit is applied to obtain the slope parameter.

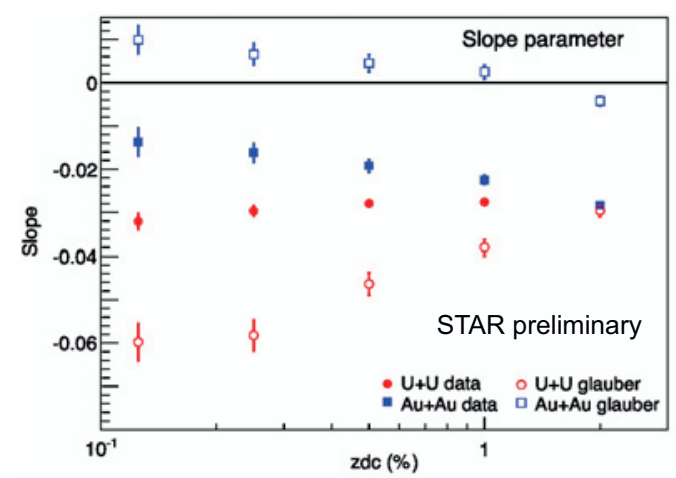

Figure 3. The slope parameters of $v_{2}$ vs. Mult $/<$ Mult $>$ in different centralities. Open symbols are from Glauber model simulation, solid symbols are from data. Blue color symbols represent the $v_{2}$ in $\mathrm{Au}+\mathrm{Au}$ collisions at $200 \mathrm{GeV}$, while $\mathrm{U}+\mathrm{U}$ data are shown in Red color.

identified particles. The $v_{2}$ of identified particles $\left(\pi^{ \pm}, K^{ \pm}, K_{S}^{0}, p, \bar{p}, \phi, \Lambda, \bar{\Lambda}, \Xi^{-}, \bar{\Xi}^{+}, \Omega^{-}, \bar{\Omega}^{+}\right)$as a function of transverse kinetic energy $m_{T}-m_{0}$ from Au+Au minimum bias, $0-30 \%$, and 30-80\% centrality at $200 \mathrm{GeV}$ are shown in Fig. 1. In minimum bias and 0-30\% centrality, the NCQ scaling holds among all particles within $10 \%$ uncertainty at $\left(m_{T}-m_{0}\right) / n_{q}>0.6 \mathrm{GeV} / c^{2}$. It indicates that the final observed $v_{2}$ is dominated by the partonic collectivity in central and minimum bias collisions. We notice that the baryon and meson start to deviate from the grouping of NCQ scaling in 30-80\% centrality. The $v_{2} / n_{q}$ of multi-strange hadrons $(\phi, \Xi)$ is lower than that of $K_{S}^{0}$ about $10-15 \%$, suggesting that multi-strange hadrons have smaller partonic collectivity in the final $v_{2}$. The measurements provide constraints to hydrodynamic model calculations when studying sQGP properties.

In year 2012 run, STAR has collected numerous data of $U+U$ collisions at $193 \mathrm{GeV}$. The uranium nucleus is much heavier than gold nucleus and larger deformation in shape. The orientations of nuclei are directly related to the initial geometry condition of the collisions. The contributions from initial geometry condition to final $v_{2}$ is quite interesting. Figure 2 shows the $v_{2}$ as a function of multiplicity divided by mean multiplicity (Mult/<Mult $>$ ) in $0.1 \%$ ZDC centrality in U+U collision at $193 \mathrm{GeV}$. The ZDC centrality is defined by the ZDC rate distribution, the ZDC rate decreases when the multiplicity become higher. The $0.1 \%$ ZDC centrality is corresponding to the most central events. It is expected that body-body collision should have less multiplicity and higher $v_{2}$, because body-body collision have larger deformation of overlap region than tip-tip collision due to the prolate shape of nuclei. The slope of $v_{2}$ as a function of Mult $/<$ Mult $>$ can be a parameter that reflects the deformation level of collisions. Figure 3 shows the slope parameter as a function of ZDC centrality in $\mathrm{Au}+\mathrm{Au}$ and $\mathrm{U}+\mathrm{U}$ collisions, both data and simulations. The slope parameters of $\mathrm{Au}+\mathrm{Au}$ and $\mathrm{U}+\mathrm{U}$ data are close to each other in peripheral collisions, and the deviation of slope parameters in two dataset starts at $1 \%$ ZDC centrality and becomes larger at more central events. The simulation with Glauber models also show similar trends and even larger deviation. The input parameters of Glauber Model are $r_{0}=6.38(6.83) \mathrm{fm}, d=0.535(0.538) \mathrm{fm}$ for gold (uranium) nucleus [8]. The slope parameter difference between $\mathrm{Au}+\mathrm{Au}$ and $\mathrm{U}+\mathrm{U}$ offers more information to the initial conditions of 


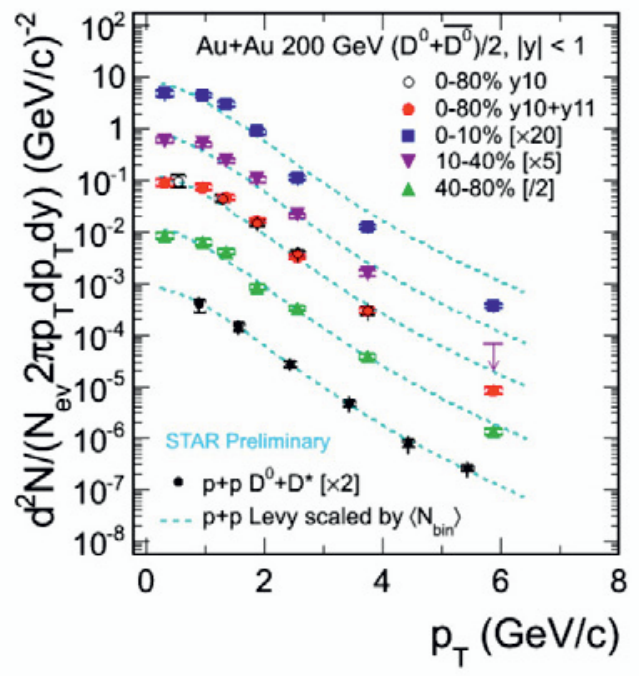

Figure 4. $D^{0}$ invariant yield as a function of $p_{T}$ in $\mathrm{p}+\mathrm{p}$ and $\mathrm{Au}+\mathrm{Au}$ collisions at $\sqrt{S_{N N}}=200 \mathrm{GeV}$. The dashed lines represent a Levi fit to $\mathrm{p}+\mathrm{p}$ data and scaled by number of binary collisions in each $\mathrm{Au}+\mathrm{Au}$ centrality bin.

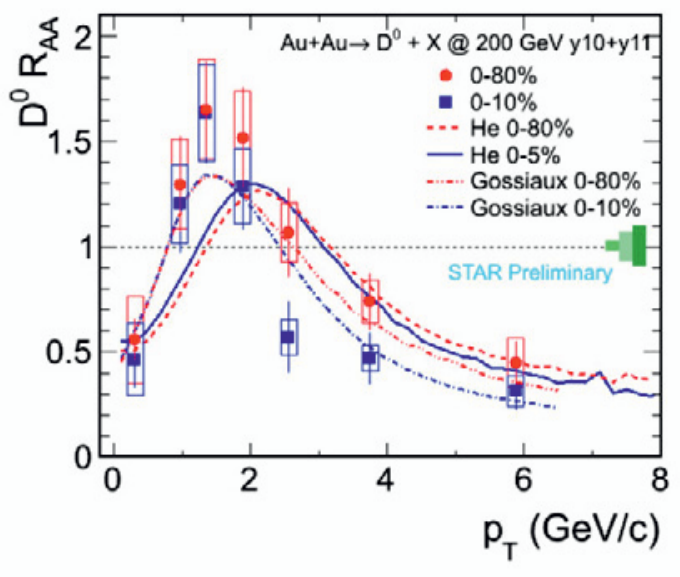

Figure 5. $D^{0}$ nuclear modification factor as a function of $p_{T}$ in central $(0-10 \%)$ and minbias $(0-80 \%) \mathrm{Au}+\mathrm{Au}$ collisions. The lines are from model predictions by $\mathrm{He}$ et al. [13] and Gossiaux et al. [14].

model calculations. Also the slope difference provide a potential way to select enhanced body-body or tip-tip collision sample which is quite interesting for initial condition study.

\section{Heavy flavor results}

STAR has measured heavy flavor production via hadronic and leptonic channels from data collected in recent years. Figure 4 shows $D^{0} p_{T}$ spectrum in $\mathrm{p}+\mathrm{p}$ and $\mathrm{Au}+\mathrm{Au}$ collisions at $\sqrt{S_{N N}}=200 \mathrm{GeV}$. The dashed lines are Levi fits to $\mathrm{p}+\mathrm{p}$ data scaled by number of binary collisions. It describes the $\mathrm{p}+\mathrm{p}$ data well. The data points lower than fits at low and high $p_{T}$, which indicates a modification of the $D^{0}$ production in Au+Au collisions. In order to quantify the modifications in different centralities, a factor called nuclear modification factor $R_{A A}$ is defined as:

$$
R_{A A}=\frac{d^{2} N_{A A} / d y d p_{T}}{N_{b i n} \times d^{2} N_{p p} / d y d p_{T}},
$$

where $d^{2} N_{A A} / d y d p_{T}$ and $d^{2} N_{p p} / d y d p_{T}$ are invariant yields in $\mathrm{Au}+\mathrm{Au}$ and $\mathrm{p}+\mathrm{p}$ collisions, respectively. $N_{\text {bin }}$ is the number of binary collisions in a given $\mathrm{Au}+\mathrm{Au}$ centrality bin. Figure 5 shows that $D^{0}$ 


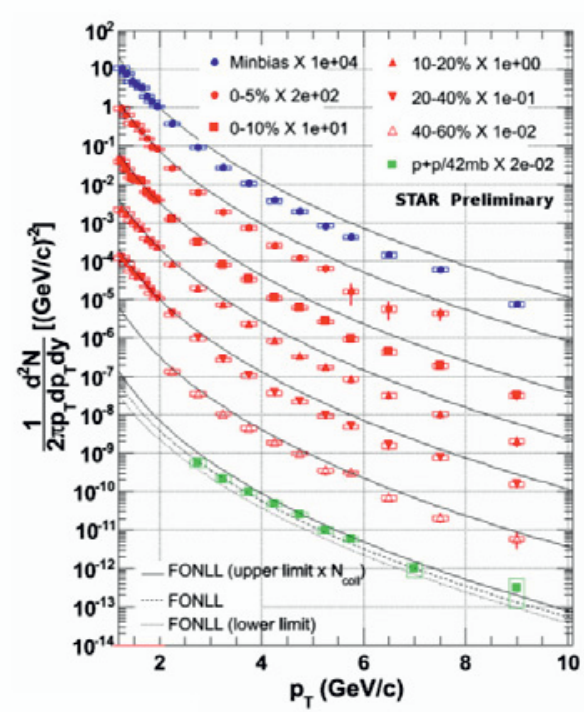

Figure 6. Invariant yields of non-photonic electrons as a function of $p_{T}$ in $\mathrm{p}+\mathrm{p}$ and $\mathrm{Au}+\mathrm{Au}$ collisions at $\sqrt{S_{N N}}=200 \mathrm{GeV}$. The solid lines represent an upper limit on FONLL predictions for NPE production scaled by number of binary collisions $\left(N_{b i n}\right)$ in a given $\mathrm{Au}+\mathrm{Au}$ centrality bin.

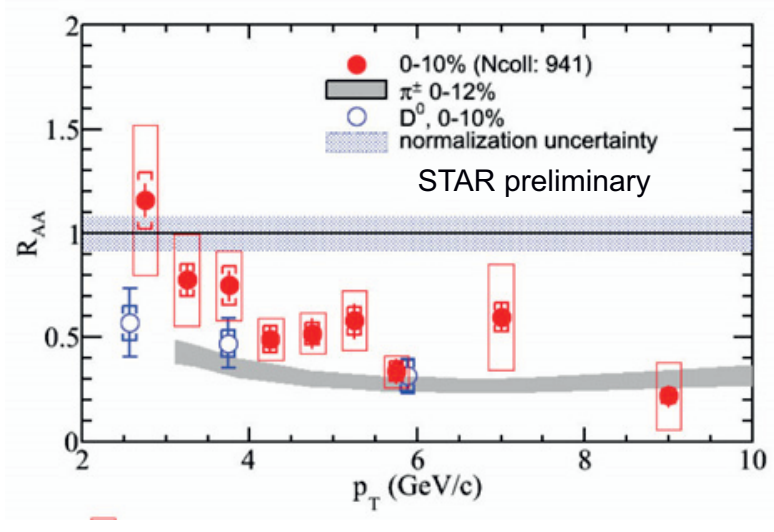

Figure 7. Non-photonic electron nuclear modification factor as a function of $p_{T}$ in central $0-10 \% \mathrm{Au}+\mathrm{Au}$ collisions.

$R_{A A}$ for $0-10 \% \mathrm{Au}+\mathrm{Au}$ most central collision is highly suppressed at low and high $p_{T}$, while appears a hump structure at intermediate $p_{T}\left(1<p_{T}<2 \mathrm{GeV} / c\right)$. The result in minbias Au+Au collisions is also shown in the Fig 4, it shows similar structure as that of central collisions but the yield suppression decreases in peripheral collisions. Two models by He et al. [13] and Gossiaux et al. [14] which assuming strongly couplings between heavy quarks and surrounding partonic medium are compared to the STAR data. Both models predict a large suppression at high $p_{T}$, and reproduce the $R_{A A}$ shape as a function of $p_{T}$. In those models, the hump at $p_{T} 1.5 \mathrm{GeV} / c$ is due to the interactions between charm quark and medium and the radial flow of light quarks which formed to D mesons with the charm quarks. The D mesons in this $p_{T}$ range are formed by coalescence approach in two models. For light hadrons (protons, pions), similar structures are observed at intermediate $p_{T}$ in $\mathrm{d}+\mathrm{Au}$, but are interpreted as Cronin enhancement [15]. The impact of Cornin enhancement as well as other cold nuclear effect on the D-meson $R_{A A}$ requires more precise measurements in peripheral $\mathrm{Au}+\mathrm{Au}$ or $\mathrm{p}(\mathrm{d})+$ Au collisions.

Another probe, electron from semi-leptonic decay of open heavy flavor meson so called nonphotonic electron (NPE), has long been proposed to be a powerful tool to study heavy flavor quark productions, although it has a mixture of electrons from charmed and bottom hadron decays. Figure 6 shows the NPE invariant yield spectra for $\mathrm{p}+\mathrm{p}$ and $\mathrm{Au}+\mathrm{Au}$ collisions at $\sqrt{S_{N N}}=200 \mathrm{GeV}$. The lines represent the FONLL calculations scaled by $N_{\text {bin }}$ corresponding to a certain centrality bin. The comparison between data and FONLL predictions shows a suppression in $\mathrm{Au}+\mathrm{Au}$ collisions. Figure 7 


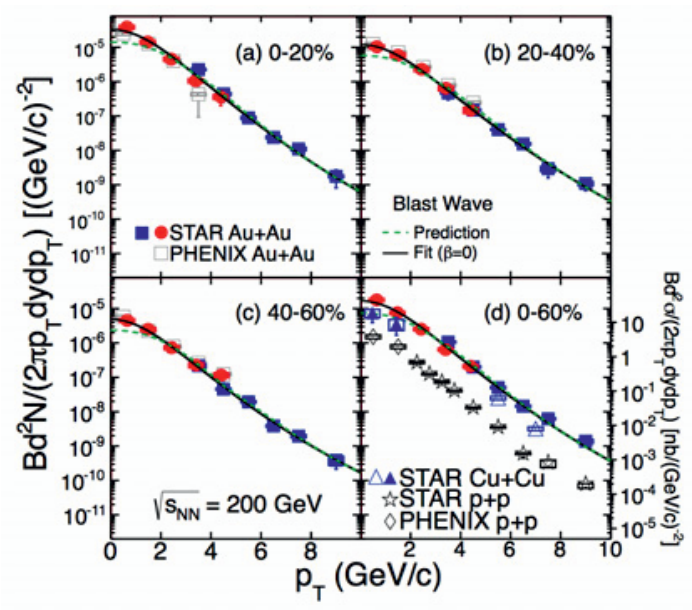

Figure 8. Invariant yields of $\mathrm{J} / \psi$ as a function of $p_{T}$ in $\mathrm{Au}+\mathrm{Au}$ collisions at $\sqrt{S_{N N}}=200 \mathrm{GeV}$. The solid lines are TBW fits with $\beta=0$. The dashed lines represents the predictions from TBW fits to other light hadrons.

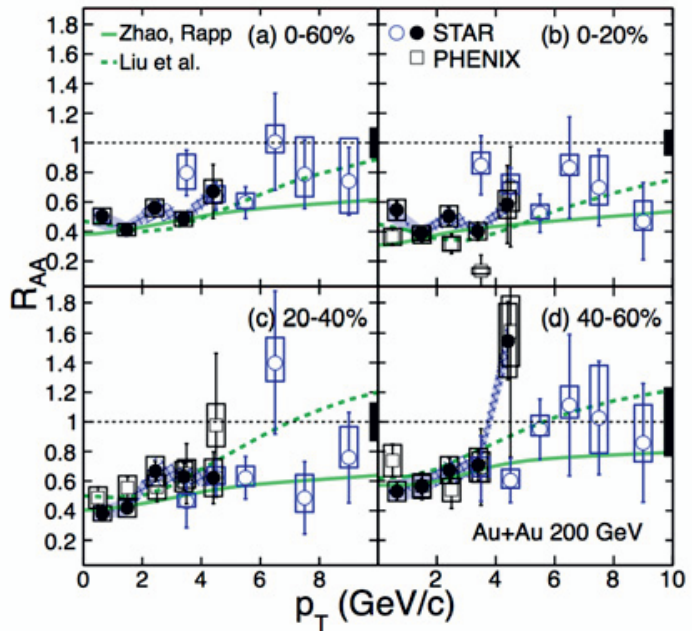

Figure 9. $\mathrm{J} / \psi R_{A A}$ versus $p_{T}$ for several centrality bins for $\mathrm{Au}+\mathrm{Au}$ collisions at $\sqrt{s_{N N}}=200 \mathrm{GeV}$. The statistical (systematic) uncertainties are shown with vertical bars (open boxes). The filled boxes about unity on the right show the size of the normalization uncertainty. The curves are from the predictions by Liu et al. [18] and Zhao et al. [19].

shows the $R_{A A}$ of NPE in most central $0-10 \% \mathrm{Au}+\mathrm{Au}$ collisions. It has strongly suppressed in central collision at high $p_{T}$, which is similar to $D^{0}$.

In addition to the open heavy flavor production, the heavy quarkonia has some unique capabilities of probing the QGP properties due to its production mechanism which is interpreted in introduction. Figure 8 shows $\mathrm{J} / \psi p_{T}$ spectra in $\mathrm{Au}+\mathrm{Au}$ collisions at $200 \mathrm{GeV}$ [16]. The dashed lines are predictions from a Tsallis Blast-Wave (TBW) model [17] with fit parameters from light hadron spectra in $\mathrm{Au}+\mathrm{Au}$ at $200 \mathrm{GeV}$. The $\mathrm{J} / \psi$ spectra at low $p_{T}$ are deviated from the TBW fits, while the solid lines show the TBW fit with a zero radial flow velocity $\beta$. The agreement between data and solid lines are reasonable well, which indicates that a small (or zero) radial flow or a significant contribution from charm quark recombination that would enhance the yield at low $p_{T}$, or both of them. Figure 9 shows the $\mathrm{J} / \psi$ $R_{A A}$ as a function of $p_{T}$ in different centralities [16]. The suppression of $\mathrm{J} / \psi$ is larger in more central collisions. For $p_{T}>5 \mathrm{GeV} / c, \mathrm{~J} / \psi R_{A A}$ becomes unity within uncertainties in the $40-60 \%$ centrality bin. The curves show two theoretical calculations $[18,19]$ including contribution from prompt production, statistical charm quark regeneration and the suppression mainly due to the color-screening effect. The models can describe data reasonably well.

In additions to the yield, the elliptic flow of $D^{0}$, NPE, and $\mathrm{J} / \psi$ are shown in Fig 10 . It shows large NPE $v_{2}$ at low $p_{T}$ which indicates strong charm-medium interactions. The $\mathrm{J} / \psi v_{2}$ at $p_{T}>2$ $\mathrm{GeV} / c$ is consistent with zero, disfavors the case that $\mathrm{J} / \psi$ is dominantly produced by coalescence from thermalized charm and anti-charm quarks at high $p_{T}$. 

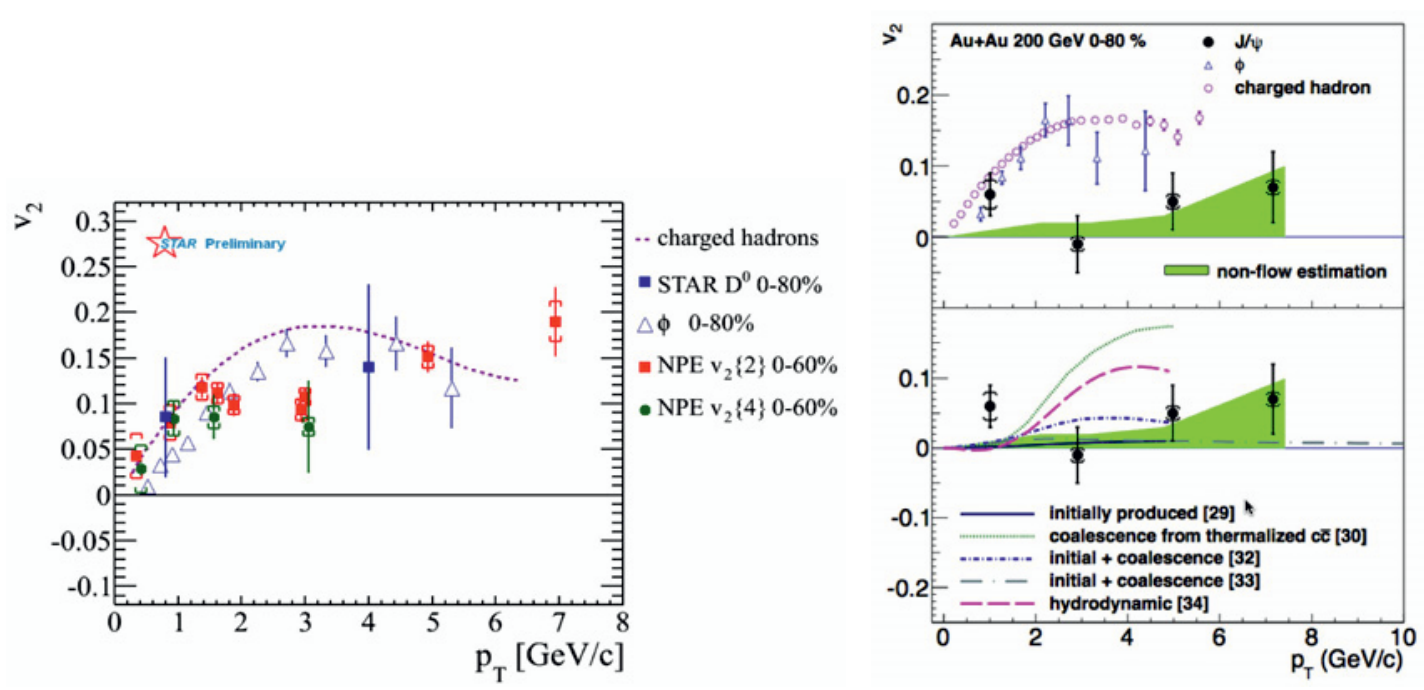

Figure 10. (Left panel) Non-photonic electron $v_{2}$ versus $p_{T}$ in minbias $\mathrm{Au}+\mathrm{Au}$ collisions at $200 \mathrm{GeV}$. (Right panel) $\mathrm{J} / \psi v_{2}$ as a function of $p_{T}$ in minbias $\mathrm{Au}+\mathrm{Au}$ collisions at $200 \mathrm{GeV}$. Band represents systematic uncertainties. Lines are model predictions [20].

\section{Dielectron measurements}

The STAR experiment has recently published dielectron measurement in $p+p$ collisions at 200 $\mathrm{GeV}$ [21]. The result serves as a solid baseline of the dielectron productions in $\mathrm{Au}+\mathrm{Au}$ collisions. The dielectron measurements within STAR acceptance $\left(p_{T}^{e}>0.2 \mathrm{GeV} / c,\left|\eta_{e}\right|<1\right.$ and $\left.\mid y_{e e}<1\right)$ in $\mathrm{Au}+\mathrm{Au}$ collisions at $200 \mathrm{GeV}$ are shown in Fig 11. A clear enhancement is observed in $\mathrm{Au}+\mathrm{Au}$ minbias and central collisions with respect to hadronic source simulations so called "cocktail" including leptonic decay channels of the $\omega, \phi$ and $J / \psi$ vector mesons as well as the Dalitz decays of the $\pi^{0}, \eta$, $\eta^{\prime}$. The $\rho$ meson contribution is not included in the cocktails. In most central Au+Au collisions, the simulation overshoots the data in IMR. It can be a possible hint of the modification of charm quarks. However, the discrepancy is still consistent within the experiment uncertainties.

Figure 12 shows the comparisons between dielectron invariant mass spectra and two model calculations: one by Rapp et al. is an effective many-body theory with broadened $\rho$ spectral function [22], the other one by Linnyk et al. is a microscopic transport model so called Parton-Hadron String Dynamics (PHSD) [23]. Rapp's calculation which describes the dimuon low mass enhancement at SPS energy very well can consistently describes the STAR data. PHSD model also well depicts the enhancement at LMR. It indicates that $\rho$ meson has a strong modification in the hot medium.

The STAR dielectron elliptic flow $v_{2}$ measurements in $\mathrm{Au}+\mathrm{Au}$ at $200 \mathrm{GeV}$ as a function of the dielectron invariant mass are presented in Fig. 13. The result shows a consistency between measurements and $v_{2}$ simulations including dielectron $v_{2}$ from $\pi^{0}, \eta, \omega$, and $\phi$ mesons. This work is still extending the measurement into intermediate mass range which will be important to disentangle the charm contributions at IMR. Dielectron measurements in both the STAR and PHENIX show a significant enhancement in LMR in heavy ion collisions at top RHIC energies [24]. It has long been considered as a result that may be connected to the chiral symmetry restoration. The dilepton measurements by the NA60 and CERES experiments are concluded that low mass enhancements at SPS 

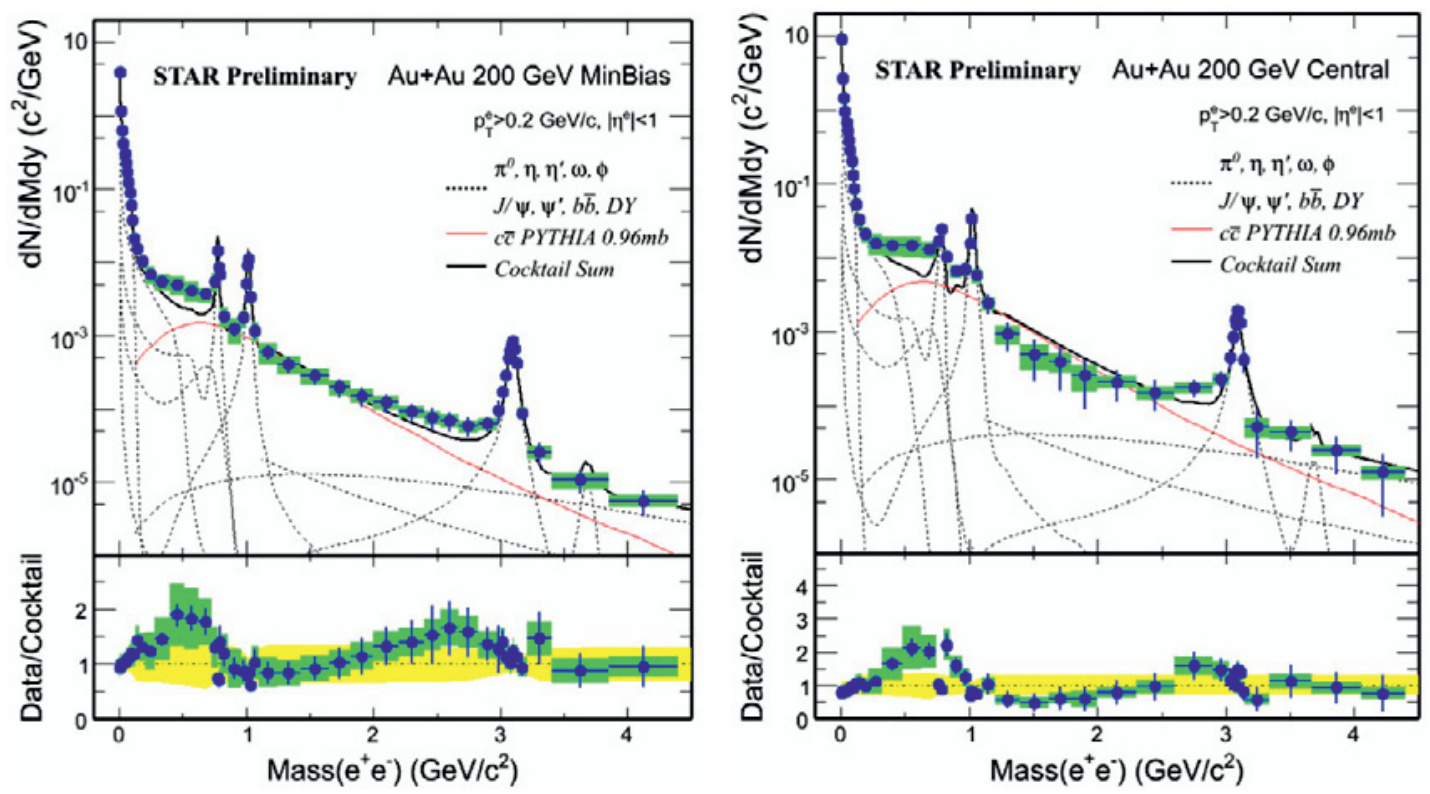

Figure 11. (Left panel) Dielectron invariant mass spectrum compares with hadronic cocktail simulation in minbias $\mathrm{Au}+\mathrm{Au}$ collisions at $200 \mathrm{GeV}$. (Right panel)Dielectron invariant mass spectrum compares with hadronic cocktail simulation in central $\mathrm{Au}+\mathrm{Au}$ collisions at $200 \mathrm{GeV}$. Hadronic cocktail simulation is shown by lines. Bars are statistical errors. Green boxes represent data systematic uncertainties and yellow band is the simulation uncertainty.
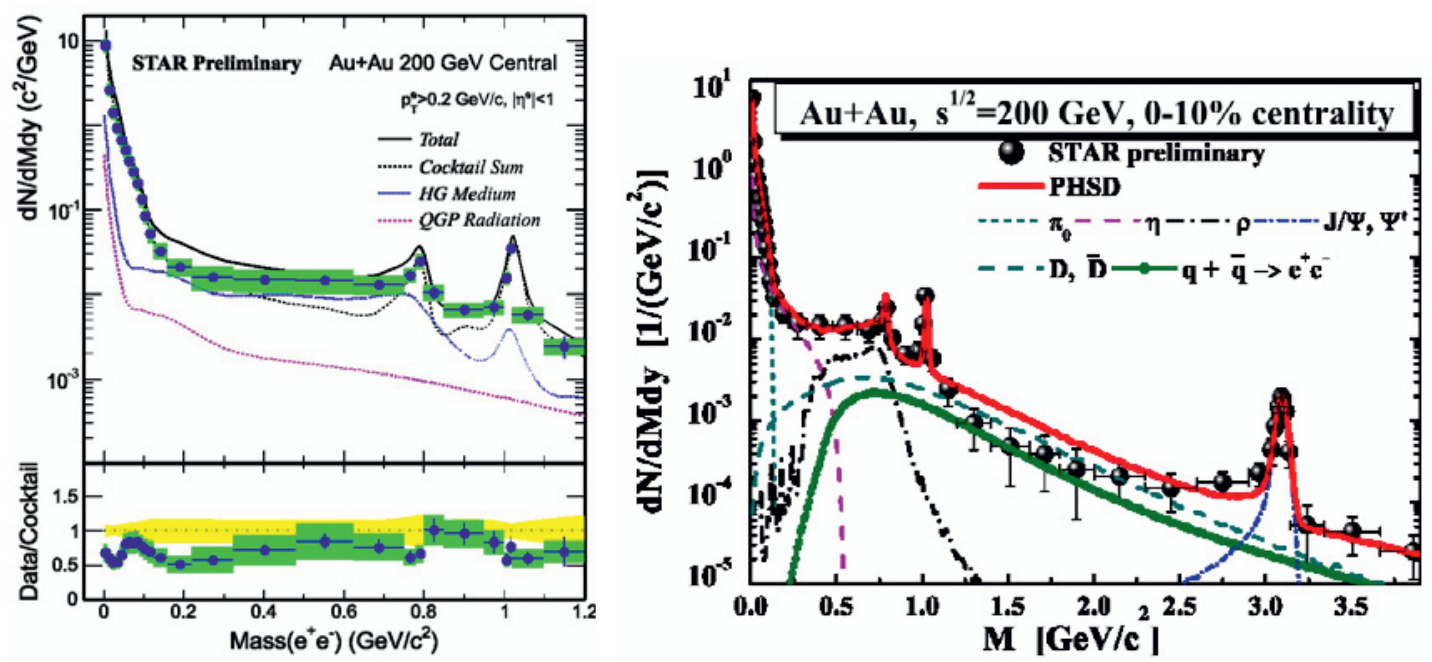

Figure 12. (Left panel) Dielectron invariant mass spectrum compares with sum of Rapp's calculation [22] and hadronic cocktail simulation in central $\mathrm{Au}+\mathrm{Au}$ collisions at $200 \mathrm{GeV}$. (Right panel) Dielectron invariant mass spectrum compares with PHSD model calculation [23] in central Au+Au collisions at $200 \mathrm{GeV}$. 


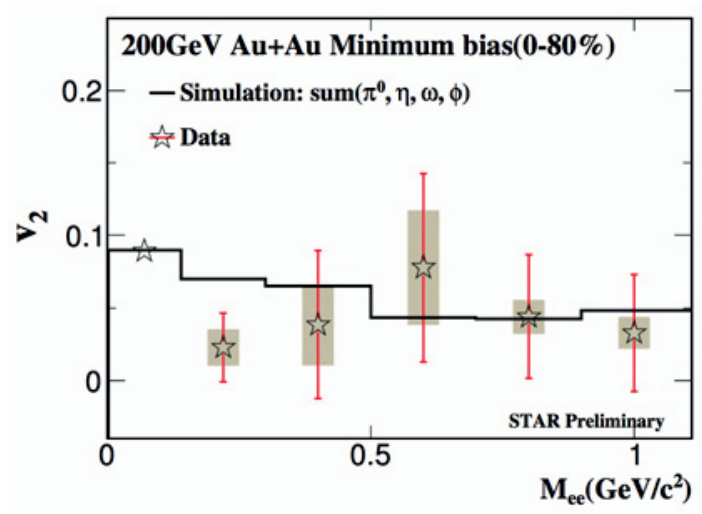

Figure 13. Dielectron elliptic flow as a function of the invariant mass in minbias Au+Au collisions at $\sqrt{s_{N N}}=200$ $\mathrm{GeV}$. The boxes indicate the systematic uncertainty. The black line is the sum of a simulation which involved $\pi^{0}$, $\eta, \omega$, and $\phi$-mesons.

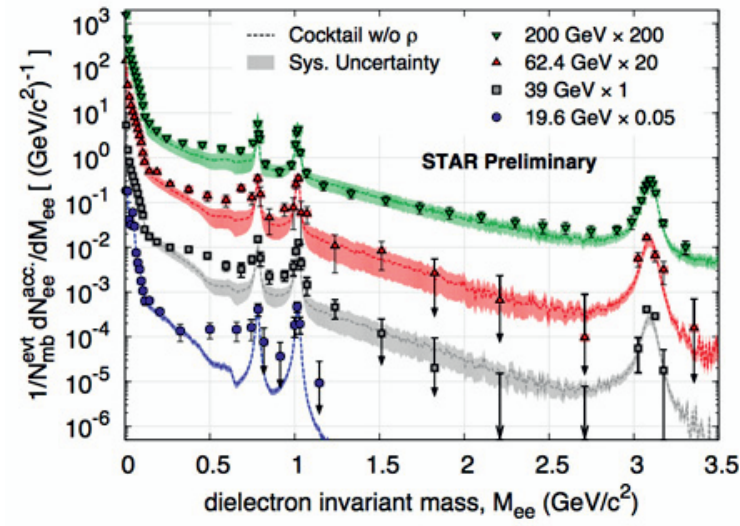

Figure 14. Dielectron invariant mass spectrum compares with hadronic cocktail simulation in $\mathrm{Au}+\mathrm{Au}$ collisions at 19.6, 39, 62.4 and $200 \mathrm{GeV}$. The dotted lines show the hadronic cocktails. The shaded areas indicate the systematic uncertainties.

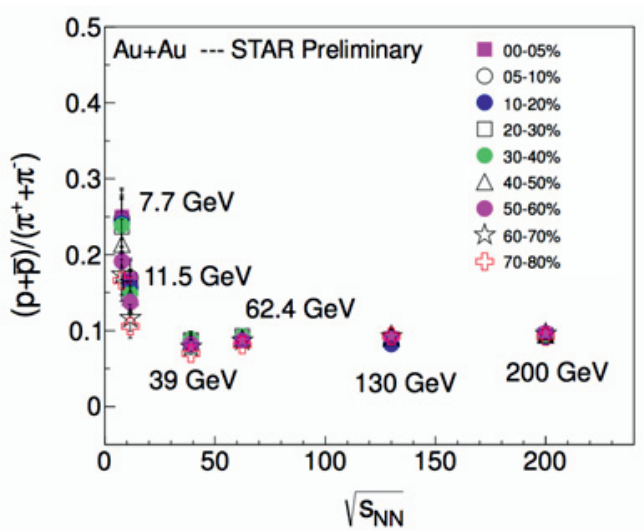

Figure 15. $(\mathrm{p}+\bar{p}) /\left(\pi^{+}+\pi^{-}\right)$in $\mathrm{Au}+\mathrm{Au}$ collisions as a function of the center-of-mass energies $\sqrt{s_{N N}}$.

energy favors a broadening of $\rho$ meson spectral shape when compared to a dropping mass scenario. The Beam Energy Scan (BES) program at RHIC provided a chance to systematically study the dielectron production from RHIC down to SPS beam energies. Figure 14 shows the inclusive dielectron invariant mass spectra within the STAR acceptance in Au+Au collisions for $\sqrt{s_{N N}}=19.6,39,62.4$, and $200 \mathrm{GeV}$, as well as the hadronic cocktail simulations. For each of the energies, a significant enhancement in LMR is observed. From the theoretical model, the in-medium $\rho$ broadening is quite related to the strong coupling to baryons, therefore the total baryon density plays an essential role in the in-medium modifications. Since the baryon-to-meson from SPS beam energy to top RHIC beam energy does not change significantly, which is shown in Fig. 15. The $\rho$ broadening scenario 
which describes SPS data well should be able to describe low mass enhancements throughout the BES energies above $19.6 \mathrm{GeV}$. Figure 16 shows comparisons between dielectron measurements and

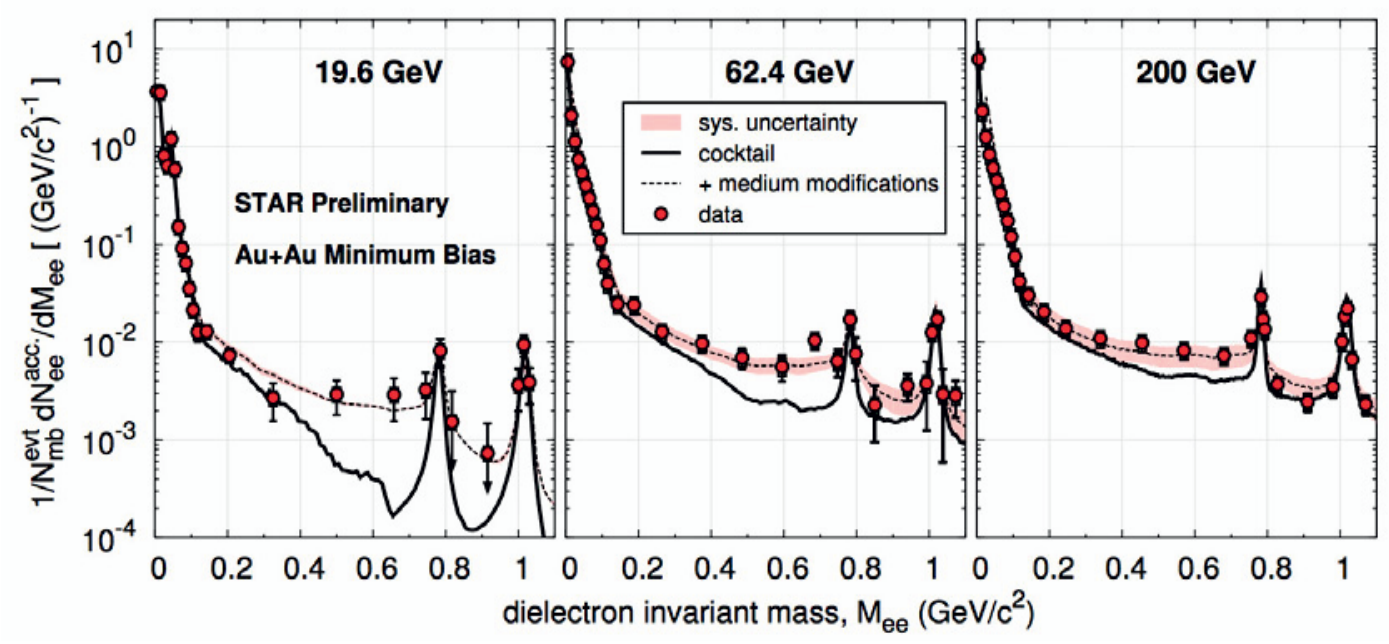

Figure 16. BES dielectron invariant-mass distributions in the low invariant-mass range from $\mathrm{Au}+\mathrm{Au}$ collisions at $19.6 \mathrm{GeV}$ (left), $62.4 \mathrm{GeV}$ (middle), and $200 \mathrm{GeV}$ (right panel). The red data points include both statistical and systematic uncertainties (boxes). The black curve depicts the hadron cocktail, while the dashed line shows the sum of the cocktail and model calculations including contributions from both the HG and the QGP phases. The systematic uncertainty on the former is shown by the light red band.

sums of hadronic cocktail simulation and theoretical calculations by Rapp [22]. In Rapp's model, the calculation includes the contribution from QGP thermal radiation and the in-medium $\rho$ meson in the hadron-gas (HG) phase which involves the broadening of $\rho$ spectra function. The comparison shows a good agreement between data and model. In future, further studies on differential measurements of the low mass dielectron distribution versus center-of-mass energy, centrality, and transverse momentum and the comparison to models will help to extensively study the vector meson spectral functions in the hadronic medium. the STAR collaboration has proposed the BES phase II program, the high statistics data will be produced at energies below $19.6 \mathrm{GeV}$ in the near future.

\section{Outlook}

STAR has stepped into the precision measurements for studies of QGP properties in recent years. We have explored the NCQ scaling for identified particles, also the initial condition of the collisions. We have measured heavy flavor production and its collectivities to reveal the interaction between heavy quark and medium. Dilepton program at STAR has achieved initial stage success of measuring dielectron in different beam energies, which are essential tools for studying the chiral symmetry restoration and QGP thermal contribution. Upcoming two major subsystem upgrades of the Heavy Flavor Tracker and the Muon Telescope Detector will significantly improve the precision of the measurements of the heavy flavor production and dileptons, and greatly help to understand sQGP properties. Both detectors are expected to be fully ready for physics in 2014 . 
ICNFP 2013

\section{References}

[1] U. W. Heinz, C. Shen and H. Song 2012 AIP Conf. Proc. 1441766

[2] J. Adams et al. 2005 Nucl. Phys. A 757102

[3] S. A. Voloshin 2005 J. Phys.: Conf. Ser. 9276

[4] M. Gyulassy and L. McLerran 2005 Nucl. Phys. A 75030

[5] P. Romatschke and U. Romantschke 2007 Phys. Rev. Lett. 99172301

[6] E. Shuryak, Phys. Lett. B78 (1978) 150.

[7] T. Matsui and H. Satz, Phys. Lett. B 178, 416 (1986); H. Satz, J. Phys. G 32, R25 (2006); A. Mocsy and P. Pe- treczky, Phys. Rev. Lett. 99, 211602 (2007); Y. Burnier, M. Laine and M. Vepsalainen, JHEP 01, 043 (2008).

[8] M. L. Miller, K. Reygers, S. J. Sanders, and P. Steinberg, Ann. Rev. Nucl. Part. Sci. 57, 205 (2007).

[9] R. Rapp and J. Wambach, Adv. Nucl. Phys. 25, 1 (2000). G. David, R. Rapp and Z. Xu, Phys. Rept. 462, 176 (2008)

[10] A. Adare et al., Phys. Rev. Lett. 104, 132301 (2010).

[11] G. Agakichiev et al., Eur. Phys. J. C 41, 475 (2005).

[12] R. Arnaldi et al., Phys. Rev. Lett. 96, 162302 (2006).

[13] M. He, R. J. Fries and R. Rapp 2012 Phys.Rev. C 86014903

[14] P. Gossiaux, J. Aichelin, T. Gousset and V. Guiho 2010 J.Phys. G 37094019

[15] A. Adare et al. (PHENIX Collaboration) 2013 (Preprint 1304.3410)

[16] L. Adamczyk et al Phys. Lett. B 722 (2013) 55

[17] Z. Tang, et al., Phys. Rev. C 79 (2009) 051901

[18] Y. Liu, Z. Qu, N. Xu, P. Zhuang, Phys. Lett. B 678 (2009) 72,

[19] X. Zhao, R. Rapp, Phys. Rev. C 82 (2010) 064905

[20] L. Adamczyk et al. Phys. Rev. Lett. 111, 052301 (2013)

[21] L. Adamczyk et al., Phys. Rev. C 86 (2012) 024906.

[22] R. Rapp, J. Wambach, H. van Hees, arXiv:0901.3289v1 [hep-ph]; R. Rapp, Phys. Rev. C 63 (2001) 054907

[23] O. Linnyk et al., Phys. Rev. C 85 (2012) 024910; H. Xu et al., Phys. Rev. C 85024906 (2012)

[24] A. Adare et al., Phys. Rev. C 81 (2010) 034911. 